\section{Dynamic properties of eye movements in mitochondrial chronic progressive external ophthalmoplegia}

${ }^{1}$ Department of Neurology, Veterans Affairs Medical Center, Case Western Reserve University, Cleveland, OH, USA

${ }^{2}$ Department of Optometry, Veterans Affairs Medical Center, Case Western Reserve University, Cleveland, OH, USA

${ }^{3}$ Department of Pathology Services, Veterans Affairs Medical Center, Case Western Reserve University, Cleveland, $\mathrm{OH}$, USA

Correspondence: RJ Leigh, Department of Neurology, 11100 Euclid Avenue, Cleveland, OH 44106-5040, USA

Tel: + 216844 3190;

Fax: + 2162313461

E-mail: rjl4@

case.edu

Received: 21 July 2007 Accepted in revised form: 2 October 2007

Published online: 26 October 2007

\begin{abstract}
Aims To relate dynamic properties of eye movements to visual disability in a patient with chronic progressive external ophthalmoplegia (CPEO) due to mitochondrial DNA deletion.

Methods Visual function was evaluated by neuro-ophthalmological examination and visual function index (VF-14). Eye movements were measured using the magnetic search coil technique.

Results The patient denied diplopia or oscillopsia at rest or during locomotion. His range of eye movements was limited to $<12$ horizontally, and $<19^{\circ}$ vertically with smaller movements of the left eye. All classes of movements were impaired, but the eyes accelerated to over $1000 \% \mathrm{~s} / \mathrm{s}$ at the onset of saccades and in response to brisk head rotations.

Conclusions Diplopia and oscillopsia were suppressed, probably due to visual system adaptation during the slow disease progression. We postulate that relative sparing of pale global extraocular fibres accounted for preserved acceleration of saccades and vestibular eye movements.

Eye (2009) 23, 382-388; doi:10.1038/sj.eye.6703024; published online 26 October 2007
\end{abstract}

Keywords: extraocular muscle; saccades; diplopia; vestibulo-ocular reflex

\section{Introduction}

Patients who present with slowly progressive limitation of eye movements and ptosis have traditionally been diagnosed with the syndrome of chronic progressive external
S DeBrosse ${ }^{1}$, EE Ubogu1 ${ }^{1}$ S Yaniglos², MO Hassan ${ }^{3}$ and RJ Leigh ${ }^{1,2}$

ophthalmoplegia (CPEO). ${ }^{1}$ Advances in genetics over the past two decades have elucidated a number of distinct diseases that may give rise to CPEO. ${ }^{1,2}$ Thus, disorders of nuclear DNA that may lead to CPEO include myotonic dystrophy, ${ }^{3}$ oculopharyngeal dystrophy, ${ }^{4}$ and other entities such as a kinship with linkage to genes concerned with heavychain myosin. ${ }^{5}$ In addition, mitochondrial myopathy resulting from a range of defects in mitochondrial DNA or mitochondrial proteinencoding nuclear DNA is often responsible for CPEO. ${ }^{2}$ Some mitochondrial DNA disorders, notably Kearns-Sayre syndrome present in childhood, ${ }^{6}$ but can usually be distinguished from congenital failure of embryogenesis of cranial nerve nuclei such as Möbius syndrome. ${ }^{7}$ Acquired disorders, especially myasthenia gravis, sometimes mimic CPEO in patients who present to physicians after the disease has been evolving for some time. ${ }^{1}$

Although the clinical features of CPEO are well described, including detailed examinations of visual function and eye movements, ${ }^{8,9}$ there are few reports of the dynamic properties of eye movements in CPEO using reliable recording techniques. We studied a patient with longstanding CPEO with the goal of defining the dynamic properties of his residual eye movements and relating them to his lack of diplopia and oscillopsia (illusory motion of the visual world) while at rest or during activities such as walking.

\section{Materials and methods}

Case report

The patient was a 67-year-old male truck driver, referred to our neurology clinic in 2004, because 
of long-standing problems with his eyes. During his fourth decade, he first became aware of mild ptosis, which was initially unilateral, but subsequently was bilateral and progressive. After about 10 years, he sought medical advice and was diagnosed with myasthenia gravis; however, the medicine prescribed did not improve his symptoms and he stopped it. He coped with his ptosis by retracting his upper eyelids with adhesive tape and continued to drive his truck. Over the subsequent seven years, he noticed progressive difficulty in shifting his direction of gaze or tracking moving objects. Although he attempted to use head movements to compensate for his difficulty with gaze control, he recognized his progressive visual disability and stopped driving a commercial vehicle. Both the patient and his wife reported that in the four years prior to our evaluation, his loss of eye movements had become worse. However, during the entire course of his illness, he had noted only occasional and fleeting diplopia. Moreover, he denied any oscillopsia at rest during walking or riding in a car.

For the prior five years, the patient also noted mild upper extremity weakness corresponding to when he started statin therapy for dyslipidaemia. Nonetheless, he had been able to continue bow-and-arrow deer hunting during the prior year. He had also sought medical treatment for hearing loss; audiometry had demonstrated some sensorineural loss in the midrange (left ear) and higher frequencies (bilateral) and he had been fitted with hearing aids. Other recent symptoms were mild memory difficulties, minor imbalance, and paresthesiae in his feet. He was receiving treatment for hypertension and had hypertrophic actinic keratosis and a resected basal cell carcinoma.

Of note was that the patient's mother, who was still alive at age 94 , had bilateral ptosis and hearing loss that had developed at about 60 years of age. His maternal aunt had also developed ptosis and subsequently underwent blepharoplasty.

Physical examination in 2007 demonstrated almost complete bilateral ptosis, even with full frontalis muscle contraction. His corrected visual acuity was 20/40 OD and $20 / 40^{-3}$ OS. Visual fields were full to confrontation. His optic fundi looked normal with no pigmentary changes. There was evidence of mild drying of the cornea, due to the patient's habit of retracting his eyelids with adhesive tape. He had bilateral cataracts, which, along with the corneal drying were sufficient to account for his vision loss. When viewing with both eyes, he denied diplopia, but stereopsis was absent (Randot Stereotest). With the Worth 4-dot test, he reported seeing five dots with his right eye, two dots straight ahead and with his left eye, three dots down and to his right.
Extraocular movements were very limited, especially in the horizontal plane. Attempts were made to estimate his range of eye movements using a Goldmann perimeter ${ }^{8}$ his right eye appeared to have $28^{\circ}$ horizontal and over $50^{\circ}$ vertical movement whereas his left eye appeared to have $22^{\circ}$ horizontal and $27^{\circ}$ vertical movement. However, the patient had difficulty in distinguishing when the visual stimulus was in his central or peripheral vision, making this approach unreliable (see measurements of eye movements in next section). Horizontal and vertical saccades were detectable at their onset, but were slow. No vergence movements could be detected. Neither attempted smooth-pursuit tracking nor head rotations increased his range of eye movements.

Although he denied oscillopsia during everyday life, when passive head rotations were induced as he viewed a rich visual background, he admitted to illusory movement of the environment (oscillopsia). In response to the visual function index (VF 14) questionnaire, ${ }^{9}$ which provides a standardized measurement of visual disability based on symptoms, he noted moderate difficulty with steps and stairs, performing handwork, and inability to read newspapers or small print; his overall score was 50 of 100 points (indicating an intermediate level of disability).

He showed no facial weakness or difficulty with speaking or swallowing and, aside from eye movements, other cranial nerves were normal. His skeletal muscle bulk, tone, and power in his limbs were judged to be normal for his age and all tendon reflexes were normal. Sensory testing and gait were also normal, including his toes.

Electrodiagnostic studies were performed in August 2005 to look for subclinical evidence of a proximal myopathy. Routine distal motor and sensory nerve conduction studies were normal. Needle electromyography of selected muscles of the left upper and lower extremities showed a mild-to-moderately severe, chronic, non-irritable proximal myopathy.

His right deltoid muscle was biopsied in August 2006. Routine light histopathology was performed on frozen and paraffin-fixed tissue. Frozen tissue was stained with hematoxylin and eosin (H\&E), myofibrillary acid and alkaline adenosine triphosphatase (ATPase), modified Gomori trichrome, nicotinamide dinucleotide tetrazolium reductase (NADH-TR), non-specific esterase, along with alkaline phosphatase, and adenylate deaminase (AMP-DA). Additional sections were stained with periodic acid Schiff (PAS) and Oil-red-O to assess glycogen and lipid stores respectively, to detect glycolytic enzymes myophosphorylase and phosphofructokinase, and to assess mitochondrial enzymes succinic dehydrogenase (SDH; Complex II) and cytochrome 
oxidase (COX; Complex IV). In addition, indirect immunohistochemistry was performed to assess for MHC class I antigen expression. Paraffin-fixed tissue specimens were stained with H\&E and Masson's trichrome. Ultrastructural examination by electron microscopy on a glutaldehyde-fixed, epoxy-resinembedded muscle specimen was also performed.

The biopsy showed mild variation in muscle fibre size with a few groups of atrophic angulated fibres. There were scattered atrophic, occasional regenerating and rare degenerating myofibres, mild endomysial fibrosis, and pyknotic nuclear clumps. There was no evidence of inflammation or vasculitis. Increased subsacrolemmal crescents were seen on oxidative stains as well as on H\&E (Figure 1a). Modified Gomori trichrome enzyme staining revealed several ragged red fibres (Figure 1b), while Toluidine blue-stained epoxy-resin-embedded sections also demonstrated increased subsacrolemmal crescents on light microscopy (Figure 1c). Scattered COXnegative fibres (within the normal limits for patient's age) were also demonstrated. There was no evidence for abnormal lipid or glycogen accumulation. Ultrastructural examination showed some myofibre Z-disk streaming, increased endomysial connective tissue with nonmembrane-bound lipid droplets, and scattered dystrophic mitochondria with electron-dense paracrystalline inclusions (Figure 1d-f). These features were consistent with a mitochondrial myopathy.

DNA was extracted from a frozen portion of the deltoid muscle biopsy to assess for mitochondrial deletions or duplications most often associated with CPEO and Kearn-Sayre Syndrome (Athena Diagnostics). Southern blot testing demonstrated a mitochondrial deletion consistent with CPEO.

\section{Eye movement measurements}

We compared our patient's eye movements with ten agematched subjects that we have previously reported. ${ }^{10}$ The patients and all control subjects gave a written informed consent in accordance with the Declaration of Helsinki
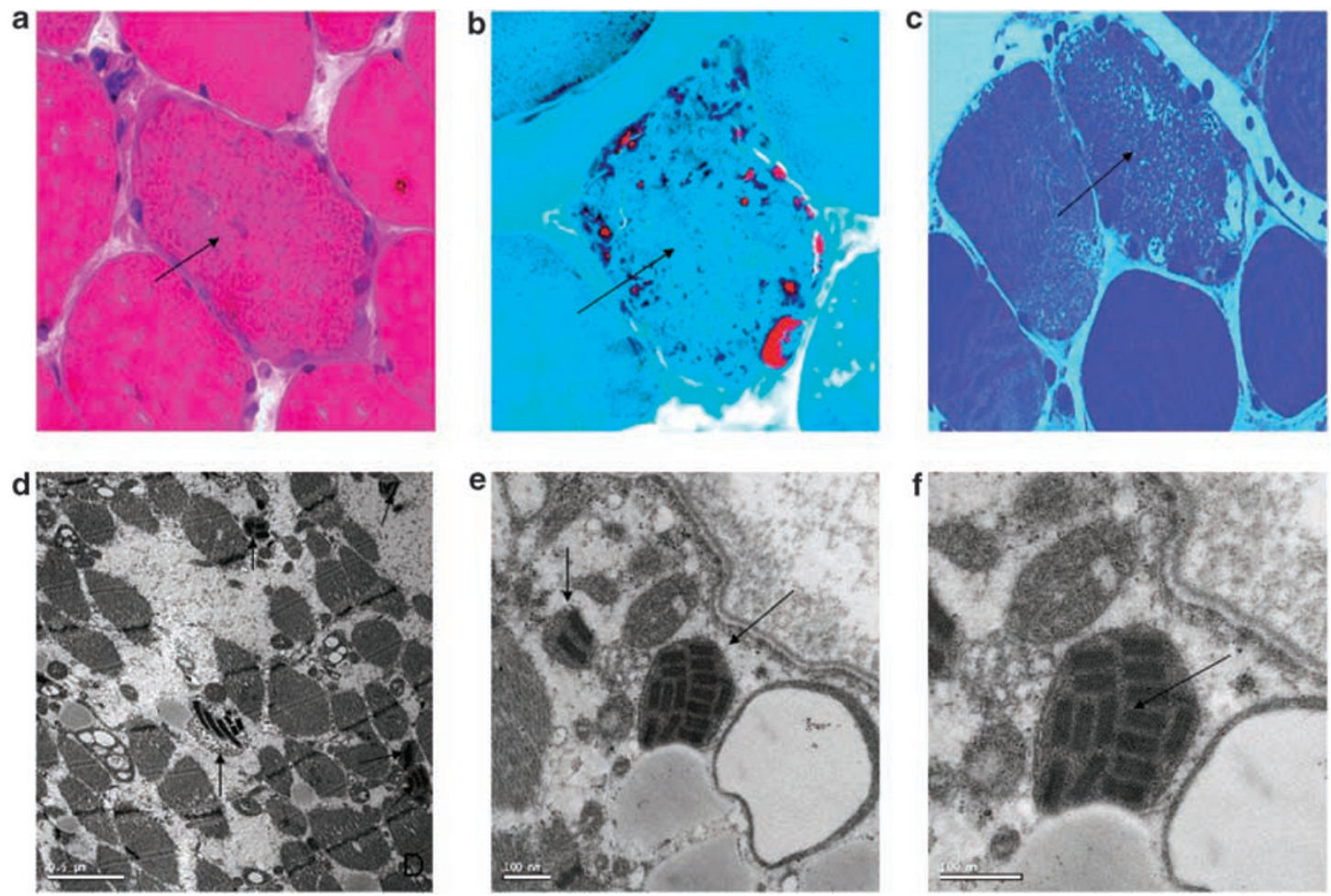

Figure 1 Pathological features of mitochondrial myopathy. Histopathological evidence for a mitochondrial myopathy is demonstrated by dystrophic myofibres (black arrows) containing subsarcolemmal accumulation of amorphous material (accumulations of abnormal mitochondria) on light microscopy stained with heamatoxylin and eosin (a), Gomori trichrome, showing the classic 'ragged red fibre' (b), and Toluidine blue (c) (all at $40 \times$ magnification). Ultrastructural examination by electron microscopy shows myofibre Z-disk streaming, increased endomysial connective tissue with non-membrane-bound lipid droplets and scattered dystrophic mitochondria with electron-dense paracrystalline inclusions (black arrows) ((d); $9400 \times$ magnification). Subsarcolemmal accumulation of normal and dystrophic mitochondria with paracrystalline inclusions (black arrows) is demonstrated ((e); $30000 \times$ magnification). A swollen dystrophic subsarcolemmal mitochondrion packed with paracrystalline inclusions (black arrows) is shown at higher power ((f); $50000 \times$ magnification). 
and the Institutional Review Board of the Cleveland Veterans Affairs Medical Center.

We measured horizontal and vertical positions of each eye using the magnetic search coil technique (CNC engineering, Seattle, WA, USA). After application of topical anaesthetic, scleral search coils were placed binocularly. The advantage of this technique is that the system is pre-calibrated and does not depend on the ability of the subject to direct gaze at visual targets. Our system provides a resolution of $0.05^{\circ}$ over a linear range of $\pm 30^{\circ}$ with a system bandwidth of $0-150 \mathrm{~Hz}$. Note that although our eye movements were precise, accuracy depends on the ability to direct each eye at a visual target, which is reliable in normal subjects but which cannot be relied on in our patient. Thus, our measurements were of eye displacements rather than absolute position, but this enabled us to reliably measure total range of movement. We tested: (1) fixation of a visual target (laser spot rear projected on a tangent screen) located at $1.2 \mathrm{~m}$; (2) saccades in response to 5-30 jumps of the visual target in the horizontal or vertical directions; (3) smooth pursuit of the visual target moving $\pm 15^{\circ}$ sinusoidally at $0.3 \mathrm{~Hz}$ horizontally or vertically; (4) vergence movements to near targets; and (5) the vestibulo-ocular reflex (VOR) during passive head rotations that were either high-acceleration step displacements (impulses) ${ }^{11}$ or sinusoidal rotations at $1-2 \mathrm{~Hz}$. Coil signals were filtered $(0-150 \mathrm{~Hz})$ prior to digitization at $500 \mathrm{~Hz}$. Eye velocity was computed as previously described; ${ }^{12}$ eye acceleration was similarly computed following low-pass digital filtering (bandwidth of $0-50 \mathrm{~Hz}$ ). Saccades onset was defined interactively, using a combination of a velocity threshold of $10^{\circ} / \mathrm{s}$ and peak acceleration. The gain of the VOR was calculated from eye velocity or head velocity. We certify that all applicable institutional and governmental regulations concerning the ethical use of human volunteers were followed during this research.

\section{Results}

During fixation of the visual target, when we covered one eye, the other made a slow but recognizable 'movement of redress.' He preferred to view with his right eye and had a left exotropia of $4.1^{\circ}$ (approximately 8 prism diopters) and left hypertropia of $2^{\circ}$ (approximately 4 prism diopters). When viewing binocularly the bright laser spot, in an otherwise dim room, he admitted to diplopia. All classes of eye movements were very limited in their range. Movements of his right eye were restricted to $11.6^{\circ}$ horizontally and $18.1^{\circ}$ vertically; and of his left eye, to $10.1^{\circ}$ horizontally and $18.8^{\circ}$ vertically. Thus, eye movements were disjunctive, more so in the horizontal plane (Figure 2). Vertical saccades (peak velocities $<65^{\circ} /$ s) tended to be faster than horizontal saccades (peak velocities $<35^{\circ} / \mathrm{s}$ ). However, although saccades were slow, the initial movement often accelerated to $>1000^{\circ} / \mathrm{s} / \mathrm{s}$, especially in his right eye (Figure 2). For normal subjects, peak acceleration of the smallest saccades usually exceeds $1000^{\circ}$ / s/s, whereas large saccades typically saturate at values of about $3500^{\circ} / \mathrm{s} / \mathrm{s}^{13}$ No smooth pursuit or vergence eye movements were evident and the patient used a series of small, slow saccades to track moving targets. In response to sinusoidal head rotations, VOR gain was estimated to be 0.07 horizontally and 0.22 vertically. In response to rapid head rotations, vestibular eye movements did not substantially increase in range (Figure 3a), but the small movements that were induced often accelerated to $>1000^{\circ}$ s s s (Figure 3b).

\section{Discussion}

We present some of the first reliable measurements of residual eye movements in CPEO due to mitochondrial myopathy. Our patient's disorder of eye movements, and ptosis developed over three decades, but was unaccompanied by complaints of diplopia or oscillopsia. Prior studies have used subjective techniques such as asking the patient to report when a small dim light, presented using a Goldmann perimeter, disappears; this point is taken as when the target has moved off the fovea and represents the extent of gaze in that direction. We used an objective method, in which a silastic scleral annulus with a fine embedded copper wire is used to signal eye rotations as the subject sits in a magnetic field. This magnetic search coil technique has now been in wide use for over 25 years and reliably measures eye rotations. When we compared the subjective Goldmann perimeter method with our search coil measurements, we found that the former gave unreliably large estimates of eye rotation. The discrepancy is probably because patients such as ours, who tolerate the visual consequences of ocular misalignment evolving over many years, cannot reliably signal the direction of a visual stimulus. Similar visual adaptation probably accounts for the lack of complaint of diplopia, except in situations such as our experimental testing (a bright laser spot in an otherwise dark room).

An unexpected finding was that although our patient's range of eye movements was very limited, he could still generate recognizable saccades. Furthermore, although the peak velocity of these saccades was $<65^{\circ} / \mathrm{s}$, the initial part of the movements accelerated the eye $>1000^{\circ} /$ $\mathrm{s} / \mathrm{s}$ (Figure 2). Thus, peak acceleration was relatively preserved compared with peak velocity, a dissociation that might seem counter-intuitive, and which is addressed further below. Impulsive head rotations 

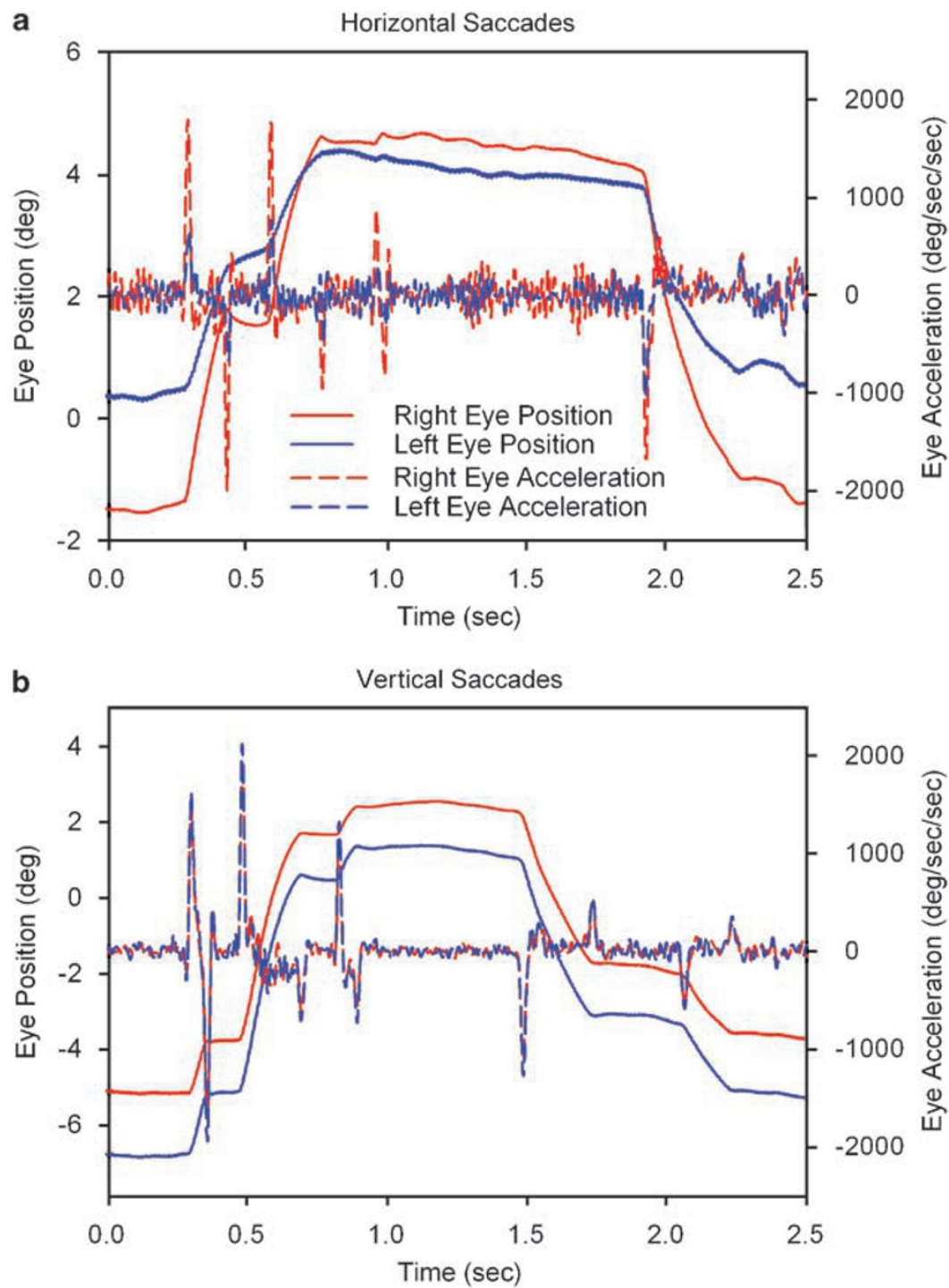

Figure 2 Representative records of horizontal (a) and vertical (b) saccades made by the patient. Note how horizontal saccades are disjunctive (larger movements of the right eye), whereas vertical movements are more conjugate. Although saccades in both directions are slow (peak velocity $<65^{\circ} / \mathrm{s}$ ), the initial accelerations especially of his right eye (see scale at right) exceed $1000^{\circ} / \mathrm{s} / \mathrm{s}$. Positive values indicate rightward or upward movements.

$>5000^{\circ} / \mathrm{s} / \mathrm{s}$ could also induce vestibular eye movements exceeding $1000^{\circ} / \mathrm{s} / \mathrm{s}$ (Figure 3), although the movements were very small. During walking, head perturbations are relatively small, but have predominant frequencies in the range $0.5-5.0 \mathrm{~Hz}^{14}$ Thus, it seems possible that the small fast movements achieved by our patient's residual VOR might at least partly compensate for the head perturbations that occur during locomotion, thereby reducing the risk of inducing oscillopsia. Overall, it appears that our patient's myopathic process led to selective sparing of small but high-acceleration eye movements. How can this finding be resolved on the basis of current concepts of mitochondrial DNA disorders affecting the extraocular muscles? $?^{2,15}$
Extraocular muscle comprises a diverse range of fibre types that are dissimilar from skeletal muscle. ${ }^{16}$ Each rectus muscle has a distinct outer orbital layer and inner global layer. In the outer orbital layer, the predominant fibre type contains a large number of mitochondria and it has been suggested that their large energy demands make them especially susceptible to the involvement in mitochondrial myopathy. ${ }^{17}$ In the inner global layer, there are a range of fibre types, including those with substantial numbers of mitochondria, but also pale fibres that are capable of fast contraction and that seem important for generating high-acceleration movements, especially saccades. ${ }^{18}$ If the major contraction of the pale global fibres occurs at the onset of saccades, as suggested 


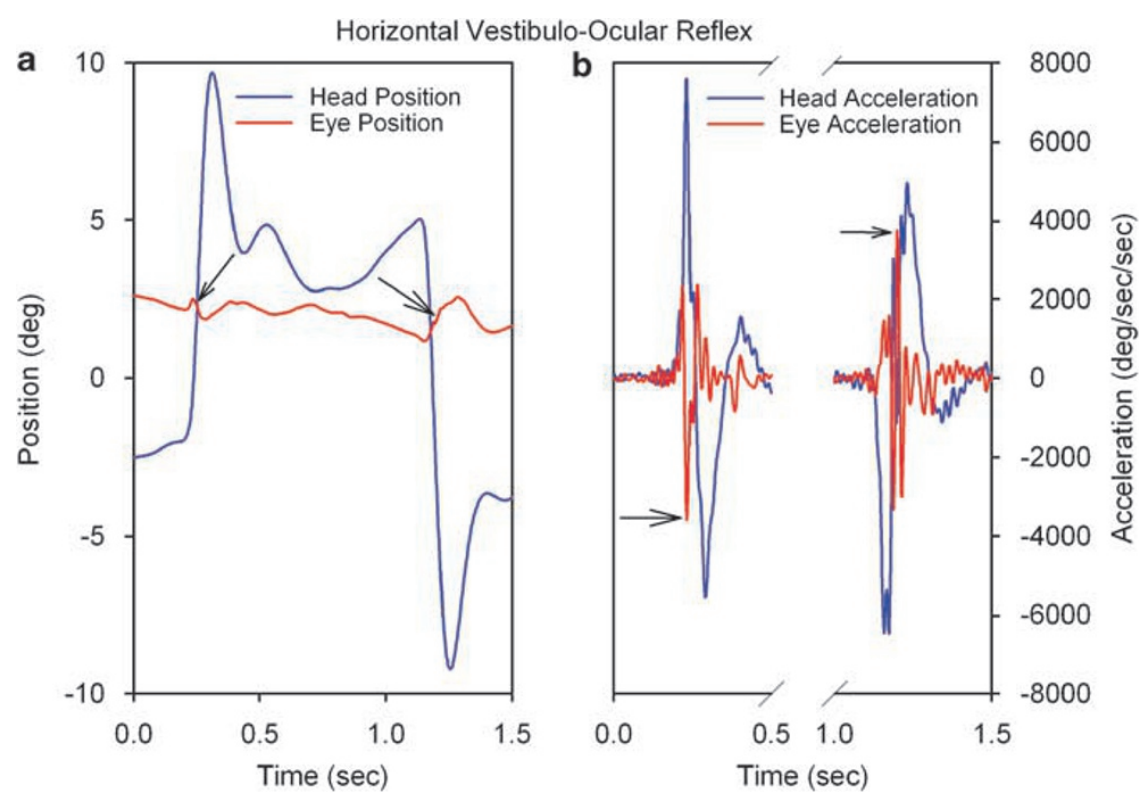

Figure 3 Representative records of the vestibulo-ocular response to rapid horizontal head rotations. In (a) an initial movement to the right induces a small eye movement to the left (first arrow) with a steep slope. The subsequent movement to the left induces a small eye movement to the right with a steep slope (second arrow). In (b) acceleration records of the same responses in (a) are shown. Although the eye movements induced by head rotation are very small (a), their peak acceleration exceeds $1000^{\circ} / \mathrm{s} / \mathrm{s}(\mathrm{b})$.

by electromyographic studies, ${ }^{18}$ then their main effect would be to overcome orbital forces that damp the onset of rapid eye movements. However, once the eye movement is started, other extraocular fibre types probably contribute to saccadic peak velocity and, in our patient, we postulate they were severely affected. In studies underway, we are testing this hypothesis using mathematical models that describe the normal behaviour of the eye muscles and fascia (ocular motor plant) during saccades. ${ }^{19}$ Do any prior reports support our hypothesis?

In a study that largely focused on saccades in myasthenia gravis, Yee $e t a^{20}$ also included a group of patients with 'progressive external ophthalmoplegia,' and measured their eye movements using electrooculography. Although these patients were reported before testing for mitochondrial DNA disorders was available, and probably included a number of diagnostic categories, one finding was that in those patients with reduced range of movement, the peak velocity of saccades did not decrease commensurately. This finding contrasted with saccades in patients with a third group of patients studied by Yee and colleagues, ocular motor nerve palsies, in which the reduction of range of movement did correlate with the amount of saccadic slowing. Interestingly, like their CPEO patients, myasthenic patients showed no correlation between restriction of range and reduced velocity. ${ }^{20}$ Preservation of saccadic acceleration in some myasthenic patients with limited range of movement has been postulated to be due to more developed post-synaptic folding at the neuromuscular junction of pale global findings compared with other fibre types. ${ }^{21}$

Our patient had mitochondrial myopathy but, as noted in the Introduction, a range of other disorders may cause the syndrome of CPEO. It would be of interest to measure the dynamic properties of eye movements in such individuals, as this would likely provide muchneeded information concerning the relationship between structure and function in disorders affecting the extraocular muscles.

\section{Acknowledgements}

Supported by the Office of Research and Development, Medical Research Service, Department of Veterans Affairs, National Institutes of Health Grant EY06717, and the Evenor Armington Fund. We are grateful to Dr John Jasper for performing the muscle biopsy.

\section{References}

1 Leigh RJ, Zee DS. The Neurology of Eye Movements (Book/ DVD). 4 edn. Oxford University Press: New York, 2006.

2 McFarland R, Taylor R, Turnbull D. The neurology of mitochondrial DNA disease. Lancet 2002; 1: 343-351.

3 Versino M, Rossi B, Beltrami G, Sandrini G, Cosi V. Ocular motor myotonic phenomenon in myotonic dystrophy. J Neurol Neurosurg Psychiatry 2002; 72: 236-240.

4 Porschke H, Kress W, Reichmann H, Goebel HH, Grimm T. Oculopharyngeal muscular dystrophy in a northern 
German family linked to chromosome $14 \mathrm{q}$, and presenting carnitine deficiency. Neuromuscul Disord 1997; 7(Suppl 1): S57-S62.

5 Lossos A, Baala L, Soffer D, Averbuch-Heller L, Dotan S, Munnich A et al. A novel autosomal recessive myopathy with external ophthalmoplegia linked to chromosome 17p13.1-p12. Brain 2005; 128: 42-51.

6 Aure K, Ogier de BH, Laforet P, Jardel C, Eymard B, Lombes A. Chronic progressive ophthalmoplegia with large-scale mtDNA rearrangement: can we predict progression? Brain 2007; 130: 1516-1524.

7 Engle EC. Oculomotility disorders arising from disruptions in brainstem motor neuron development. Arch Neurol 2007; 64: 633-637.

8 Richardson C, Smith T, Schaefer A, Turnbull D, Griffiths P. Ocular motility findings in chronic progressive external ophthalmoplegia. Eye 2005; 19: 258-263.

9 Yu Wai Man CY, Smith T, Chinnery PF, Turnbull DM, Griffiths PG. Assessment of visual function in chronic progressive external ophthalmoplegia. Eye 2006; 20: 564-568.

10 Kumar AN, Han YH, Liao K, Rucker JC, Ramat S, Leigh RJ Evaluating large saccades in patients with brain-stem or cerebellar disorders. Ann N Y Acad Sci 2005; 1039: 404-416.

11 Halmagyi GM, Curthoys IS. A clinical sign of canal paresis. Arch Neurol 1988; 45: 737-739.
12 Ramat S, Somers JT, Das VE, Leigh RJ. Conjugate ocular oscillations during shifts of the direction and depth of visual fixation. Invest Ophthalmol Vis Sci 1999; 40: 1681-1686.

13 Ramat S, Das VE, Somers JT, Leigh RJ. Tests of two hypotheses to account for different-sized saccades during disjunctive gaze shifts. Exp Brain Res 1999; 129: 500-510.

14 Grossman GE, Leigh RJ, Abel LA, Lanska DJ, Thurston SE. Frequency and velocity of rotational head perturbations during locomotion. Exp Brain Res 1988; 70: 470-476.

15 Taylor RW, Turnbull DM. Mitochondrial DNA mutations in human disease. Nat Rev Genetics 2005; 6: 389-402.

16 Spencer RF, Porter JD. Biological organization of the extraocular muscles. Prog Brain Res 2006; 151: 33-79.

17 Yu Wai Man CY, Chinnery PF, Griffiths PG. Extraocular muscles have fundamentally distinct properties that make them selectively vulnerable to certain disorders. Neuromuscul Disord 2005; 15: 17-23.

18 Scott AB, Collins CC. Division of labor in human extraocular muscle. Arch Ophthalmol 1973; 90: 319-322.

19 Zee DS, FitzGibbon EJ, Optican LM. Saccade-vergence interactions in humans. J Neurophysiol 1992; 68: 1624-1641.

20 Yee RD, Whitcup SM, Williams IM, Baloh RW, Honrubia V. Saccadic eye movements in myasthenia gravis. Ophthalmology 1987; 94: 219-225.

21 Khanna S, Liao K, Kaminski HJ, Tomsak RL, Joshi A, Leigh RJ. Myasthenia revisited: new insights from pseudointernuclear ophthalmoplegia. J Neurol 2007; e-pub ahead of print 27 August 2007. 